\title{
Optical coherence tomography angiography of foveal hypoplasia
}

Kaivon Pakzad-Vaezi, MD1, Pearse Keane, MD ${ }^{1,2}$, João Nobre Cardoso, MD, Catherine Egan, MD¹, Adnan Tufail, MD¹.

Moorfields Eye Hospital NHS Foundation Trust, Department of Medical Retina, 162

City Road, London, United Kingdom, EC1V 2PD

2Institute of Ophthalmology, University College London, 11-43 Bath St, London,

United Kingdom, EC1V 9EL

Corresponding Author:

Kaivon Pakzad-Vaezi, MD

Department of Medical Retina

Moorfields Eye Hospital

162 City Road

London, United Kingdom

EC1V 2PD

Email: kpv@alumni.ubc.ca

Telephone: +4402075662576

Fax: +440207521 4699

Sub-title:

Foveal hypoplasia demonstrates no foveal avascular zone in the superficial capillary plexus as imaged by optical coherence tomography angiography, while a partial one exists in the deep capillary plexus, having potential implications for foveal development.

Word Count: 1281

Figures: 3

Tables: 1

References: 15 


\begin{abstract}
Aims: To discuss foveal development in the context of detailed retinal vasculature imaging in foveal hypoplasia using optical coherence tomography angiography.
\end{abstract}

Methods: In this case series, the optical coherence tomography angiography results of four patients with idiopathic foveal hypoplasia, and two patients with foveal hypoplasia secondary to oculocutaneous albinism are presented.

Results: Cases with intact visual acuity demonstrated lower grades of foveal hypoplasia on optical coherence tomography, while those with poor vision demonstrated high grades of foveal hypoplasia. The superficial retinal capillary plexus was intact in the foveal area in all cases, with no demonstrable foveal avascular zone. The deep retinal capillary plexus was absent to variable degrees in most cases, but was most persistent in those cases with reduced vision.

Conclusion: The superficial retinal capillary plexus is present in cases with foveal hypoplasia, while the deep retinal capillary plexus is absent to varying degrees. Our findings support the hypothesis that an intact foveal avascular zone of the deep capillary plexus allows for outer retinal photoreceptor specialization to occur unimpeded, resulting in preserved visual acuity, while this process may be inhibited by an absent deep capillary foveal avascular zone with resultant poor vision. 
Introduction

Foveal hypoplasia, defined as an underdeveloped fovea and usually associated with reduced vision, is seen in conditions such as aniridia, albinism, nanophthalmos, incontinentia pigmenti, retinopathy of prematurity, and achromatopsia.[1-3] It can also be an isolated cause of poor vision and nystagmus.[4, 5] Imaging techniques have demonstrated the persistence of inner retinal layers and absence of a structural pit in foveal hypoplasia on optical coherence tomography (OCT), and absence of the foveal avascular zone (FAZ) on fluorescein angiography (FA).[1] Importantly, visual acuity can be preserved despite these anatomical findings, and has been termed fovea plana.[1, 6]

The advent of optical coherence tomography angiography (OCTA) has allowed for the non-invasive detailed analysis of retinal vasculature stratified by layer.[7] When presented en face, OCTA images mimic traditional FA images. A significant advantage over FA, however, is the ability of OCTA to delineate specific vascular layers with minimal artifact heretofore poorly imaged by FA, specifically the deep capillary plexus, the radial peripapillary network, and the choriocapillaris.[7]

Using OCTA, we present cases of foveal hypoplasia in patients with reduced and intact visual acuity. 
Materials and Methods

In this retrospective case series, electronic chart review of patients presenting to Moorfields Eye Hospital's medical retina service were analyzed for the diagnosis of foveal hypoplasia. If OCTA was performed as part of their routine imaging workup, their anonymous data was included in this study, including demographic information, diagnosis, visual acuity, OCT results, electrodiagnostic reports, and OCTA images. This study received approval by the Moorfields Eye Hospital ethical review board.

The Optovue RTVue XR Avanti (Optovue, Inc., Fremont, CA, USA) was used to obtain OCTA images. The details of this imaging device are reviewed elsewhere.[7] The standard images obtained included $3 \mathrm{~mm} \times 3 \mathrm{~mm}$ and $8 \mathrm{~mm} \times 8 \mathrm{~mm}$ macular scans requiring excellent fixation, with multiple attempts in various positions of gaze required in those with nystagmus. Standard instrument segmentation parameters were used to define superficial and deep capillary plexi, specifically from $3 \mu \mathrm{m}$ below the internal limiting membrane to $15 \mu \mathrm{m}$ below the internal plexiform layer (IPL) for the former, and between 15 and $70 \mu \mathrm{m}$ below the IPL for the latter. Manual segmentation was used to confirm that the presence or absence of the FAZ was not due to segmentation error. Macular OCT scans were obtained with the Spectralis HRA+OCT (Heidelberg Engineering; Heidelberg, Germany).

Degrees of foveal hypoplasia were defined according to the OCT grading system proposed by Thomas et al.[8] In this system, a normal fovea is defined by the absence of plexiform layers, the presence of a foveal pit, the presence of outer segment lengthening, and the presence of outer nuclear layer (ONL) widening. 
Grade 1 foveal hypoplasia lacks the first feature of an otherwise normal fovea. Grade 2 lacks the first two features. Grade 3 lacks the first three features, while Grade 4 lacks all features. Increasing grades of foveal hypoplasia were correlated with worsening visual acuity.[8]

Results

Seven patients met inclusion criteria (Table). 


1
2
3
4
$5^{*}$
6

$\begin{array}{lc}43 / \mathrm{F} & \text { Idiopathic } \\ 59 / \mathrm{F} & \text { Idiopathic } \\ 14 / \mathrm{F} & \text { Idiopathic } \\ 60 / \mathrm{M} & \text { Idiopathic } \\ 81 / \mathrm{F} & \text { Oculocutaneous albinism } \\ 40 / \mathrm{M} & \text { Oculocutaneous albinism }\end{array}$

B: $6 / 6$

R: 6/9; L: 6/12

B: $6 / 15$

Normal

R: 6/12; L: 6/24

B: $6 / 18$

B: $6 / 60$

No
No
No
No
Yes
Yes

Normal

Normal

Normal

$\begin{array}{ll}\text { No } & 1 \\ \text { No } & 1 \\ \text { No } & 3 \\ \text { No } & 3 \\ \text { Yes } & 3 \\ \text { Yes } & 4\end{array}$

No

Table. Characteristics of six patients with foveal hypoplasia

pERG: pattern eletroretinogram. VEP: visual evoked potential. OCT: optical coherence tomography. FAZ: foveal avascular zone. OCTA: optical coherence tomography angiography. R: right eye. L: left eye. B: each eye individually. NA: not available. *Patient 5 also demonstrated RPE changes associated with dry age-related macular degeneration in both eyes, and a mild epiretinal membrane in the left eye. 
One patient with ocular albinism was excluded due to inadequate image quality secondary to poor fixation. Ages ranged from 14 to 81, with two males. Four cases were idiopathic, while two had a diagnosis of oculocutaneous albinism (OCA). Foveal hypoplasia was an incidental finding in all idiopathic cases, all of who were born at term. Visual acuities for the idiopathic cases ranged from 6/6-6/24, with no cases exhibiting nystagmus, pattern electroretinography (pERG) abnormalities, or intracranial misrouting on pattern visual evoked potential (VEP) testing. All albinism cases demonstrated nystagmus and intracranial misrouting on VEP. The two OCA cases had bilateral visual acuities of $6 / 60$ and $6 / 18$, respectively. Idiopathic cases demonstrated lower grades of foveal hypoplasia on OCT, while OCA cases demonstrated higher grades. Cases with intact or mildly reduced vision demonstrated grade 1 hypoplasia, moderate visual loss was associated with grade 3 hypoplasia, whilst severe loss of vision demonstrated grade 4 hypoplasia (Figure 1; Table).

On OCTA, no specific abnormalities were noted in the outer retina or choriocapillaris (Figures 2,3). The outer retina was avascular in all cases. There was no evidence of a FAZ in the superficial plexus. However, there was a small or partial FAZ in the deep plexus in all cases. 
Discussion

Foveal development begins at fetal week 12, with a distinct pit first visualized between week 25-28.[9] Centripetal displacement of all retinal layers begins prior to pit formation and is the predominant process before birth, resulting in both cone packing and retinal thickening.[6,10] At term birth, both centripetal displacement of cones and centrifugal displacement of inner retinal layers are occurring, resulting in further cone packing and pit formation. $[6,9,10]$ The fovea continues to grossly develop by postnatal 3.8 years, becoming more shallow and wide with ocular growth. Cone packing, however, continues to occur into adolescence.[6, 9, 10]

The FAZ is thought to be critical in the development of the foveal pit. Previously thought to be vascularized at some point, more convincing evidence indicates that the FAZ is always avascular during development. $[6,11]$ When it fails to form, the foveal pit also fails to form.[11-13] In addition, a larger FAZ is associated with a larger foveal pit.[3] A distinct FAZ has been reported as early as gestational age 25 weeks.[14] Prenatally, local factors from ganglion cells prevent astrocyte and endothelial cell ingrowth into the future foveola and help guide its development.[6] Lacking structural support from a vascular network, the fovea forms a pit secondary to intraocular pressure mechanical forces, while inner retinal cells are able to spread outwards unimpeded by vessels. $[6,13]$

Neither the FAZ nor the foveal pit appears to be prerequisite for intact vision. We have shown cases of both poor and intact visual acuity being compatible with a 
vascularised fovea. Marmor et al[1] demonstrated near normal vision in adults without a foveal pit or FAZ, along with intact multifocal electroretinography and normal outer retina structural cone specialization on OCT and adaptive optics. Noval et al[2] demonstrated normal or near normal vision in children lacking foveal pits, but intact outer retinal parameters. Indeed, as delineated by Thomas and coworkers' grading scheme, milder degrees of foveal hypoplasia are compatible with better vision.[8] Importantly, visual acuity suffers when outer retinal cone specialization (packing; outer segment lengthening) fails, as opposed to inner retinal pit formation. $[3,6,8]$

Our OCTA findings are compatible with the above discussion. Centripetal displacement of cones begins prior to pit formation or FAZ completion, indicating their independence. Centrifugal displacement of inner retinal layers and pit formation, however, do not occur in the vascular fovea. This is thought to be due to increased retinal structural rigidity resisting mechanical pit deformation from intraocular pressure, and difficulty of inner retinal horizontal displacement in the presence of retinal vasculature. The outer retina, confirmed to be avascular on OCTA despite lacking a FAZ, offers no resistance to continued centripetal cone movement and specialization, resulting in intact vision. Cases with a more defined deep capillary FAZ tended to demonstrate better visual acuity, while the two OCA cases demonstrated a more vascularized deep plexus, possibly contributing to the inhibition of outer retinal specialization and resultant poorer vision. Further work is required to elucidate which factors are involved in outer retinal packing and specialization. Furthermore, a deep capillary FAZ on OCTA may reflect the normal 
anastomosis of this vasculature to the superficial plexus, which occurs on the foveal slope in macaque monkeys.[15] Further analysis with 3D rendering may address this issue.

In summary, OCTA imaging in patients with foveal hypoplasia shows persistence of the superficial retinal capillary plexus at the fovea, but absence of the deep retinal capillary plexus in those with intact visual acuity. During development, this outer retinal foveal avascularity may allow continued centripetal cone movement and specialization, and thus allow for relative preservation of good visual acuity. 


\section{Acknowledgements}

Betty MacPhee: image collection.

Competing interests:

KPV: none.

PK: speaker fees for Heidelberg, Topcon, Bayer, Allergan, Novartis

JNC: none.

CE: advisory boards for Heidelberg, Novartis, Haag-Streit

AT: advisory boards for Heidelberg and Optovue

Author contributions:

KPV: acquisition, analysis, or interpretation of data; drafting of the manuscript; administrative, technical, or material support.

PK: conception or design; critical revision of the manuscript for important intellectual content; supervision.

JNC: acquisition, analysis, or interpretation of data; critical revision of the manuscript for important intellectual content

CE: conception or design; critical revision of the manuscript for important intellectual content; supervision.

AT: conception or design; critical revision of the manuscript for important intellectual content; supervision.

Funding:

None 
References:

1. Marmor MF, Choi SS, Zawadzki RJ, et al. Visual insignificance of the foveal pit: reassessment of foveal hypoplasia as fovea plana. Arch Ophthalmol 2008;126:90713.

2. Noval S, Freedman SF, Asrani S, et al. Incidence of fovea plana in normal children. J AAPOS 2014;18:471-5.

3. Dubis AM, Hansen BR, Cooper RF, et al. Relationship between the foveal avascular zone and foveal pit morphology. Invest Ophthalmol Vis Sci 2012;53:1628-

36.

4. Curran RE, Robb RM. Isolated foveal hypoplasia. Arch Ophthalmol 1976;94:48-50.

5. Thomas MG, Gottlob I. Optical coherence tomography studies provides new insights into diagnosis and prognosis of infantile nystagmus: a review. Strabismus 2012;20:175-80.

6. Provis JM, Dubis AM, Maddess T, et al. Adaptation of the central retina for high acuity vision: cones, the fovea and the avascular zone. Prog Retin Eye Res 2013;35:63-81.

7. Spaide RF, Klancnik JM, Cooney MJ. Retinal vascular layers imaged by fluorescein angiography and optical coherence tomography angiography. JAMA Ophthalmol 2015;133:45-50.

8. Thomas MG, Kumar A, Mohammad S, et al. Structural grading of foveal hypoplasia using spectral-domain optical coherence tomography a predictor of visual acuity? Ophthalmology 2011;118:1653-60.

9. Hendrickson A, Possin D, Vajzovic L, et al. Histologic development of the human fovea from midgestation to maturity. Am J Ophthalmol 2012;154:767-78. 10. Lee H, Purohit R, Patel A, et al. In Vivo Foveal Development Using Optical Coherence Tomography. Invest Ophthalmol Vis Sci 2015;56:4537-45.

11. Provis JM, Hendrickson AE. The foveal avascular region of developing human retina. Arch Ophthalmol 2008;126:507-11.

12. Springer AD, Hendrickson AE. Development of the primate area of high acuity. 1. Use of finite element analysis models to identify mechanical variables affecting pit formation. Vis Neurosci 2004;21:53-62.

13. Springer AD, Hendrickson AE. Development of the primate area of high acuity, 3: temporal relationships between pit formation, retinal elongation and cone packing. Vis Neurosci 2005;22:171-85.

14. Hughes S, Yang H, Chan-Ling T. Vascularization of the human fetal retina: roles of vasculogenesis and angiogenesis. Invest Ophthalmol Vis Sci 2000;41:121728.

15. Provis JM, Sandercoe T, Hendrickson AE. Astrocytes and blood vessels define the foveal rim during primate retinal development. Invest Ophthalmol Vis Sci 2000;41:2827-36. 
Figure 1.

Optical coherence tomography of six patients with different grades of foveal hypoplasia (FH). A-F. Patients with FH. a-f. Corresponding age-matched normal foveal scans. A. Patient 1 with Grade 1 FH. B. Patient 2 with Grade 1 FH. C. Patient 3 with Grade 3 FH. D. Patient 4 with Grade 3 FH. E. Patient 5 with Grade 3 FH and RPE changes associated with dry age-related macular degeneration. F. Patient 6 with Grade 4 FH. A-D: idiopathic foveal hypoplasia. E-F: oculocutaneous albinism. Grades of FH sequentially include persistence of the plexiform layers at the fovea (Grade 1, arrows), absence of the foveal pit (Grade 2, single asterisk), absence of outer segment lengthening (Grade 3, arrowheads), and absence of outer nuclear layer widening (Grade 4, double asterisk). Corresponding lack of FH signs shown in panel a.[8]

Figure 2.

Optical coherence tomography angiography images demonstrating superficial $(\mathrm{A}, \mathrm{C})$ and deep (B, D) vascular plexi in the right eye of Patient 1 (A, B; 8 x $8 \mathrm{~mm}$ ) and left eye of Patient 2 (C, D; $3 \times 3 \mathrm{~mm}$ ). Age- and gender-matched normal controls indicated by corresponding lower case letters (superficial plexi: $a, c$; deep plexi: b, d). Definite foveal avascular zones indicated by arrows. Control 1 (a, b; 8 x 8 mm): left eye. Control 2 (c, d; 3 x 3 mm): right eye.

Figure 3.

Optical coherence tomography angiography images demonstrating superficial $(A, C)$ and deep (B, D) vascular plexi in the right eyes of Patients 4 (A, B; $3 \times 3 \mathrm{~mm})$ and 5 (C, D; $3 \times 3 \mathrm{~mm}$ ). Age- and gender-matched normal controls indicated by corresponding lower case letters (superficial plexi: a, c; deep plexi: b, d). Definite foveal avascular zones (FAZ) indicated by arrows; partial FAZ indicated by asterisk. Control 4 (a, b; 3 x 3 mm): right eye. Control 5(c, d): right eye. 
